\title{
BMC Dermatology reviewer acknowledgement, 2014
}

Guangde Tu

\section{Contributing reviewer}

The editors of BMC Dermatology would like to thank all our reviewers who have contributed their time to the journal in Volume 14 (2014).

\author{
Turkey \\ Isabel Andia \\ Spain \\ Zoe Apalla \\ Greece
}

Ilknur Altunay

Alejandro Arenas-Pinto
United Kingdom

Giuseppe Argenziano

Italy

Wioletta Baranska-Rybak

Poland

Ioannis Bassukas

Greece

Robert Bransfield

USA

Robert Brodell

USA

Alex Burdorf

Netherlands

Ian Burgess

United Kingdom

Marco Castori

Italy

Hector Chinoy

United Kingdom

\author{
Elliot Coups \\ USA
}

Marian Currie

Australia

Soheil Dadras

USA

Robert Dellavalle

USA

Hoelzle Erhard

Germany

Stefano Fedele

United Kingdom

Steven Feldman

USA

Noriki Fujimoto

Japan

Georgios Gaitanis

Greece

Rebat Halder

USA

Pilar Iranzo

Spain

Takamichi Ito

Japan

Munenari Itoh

Japan
Feroze Kaliyadan

Saudi Arabia

Grazyna Kaminska-Winciorek

Poland

Jennifer Koplin

Australia

Chembolli Lakshmi

India

Peter Lio

USA

Marie Loden

Sweden

Ingrid Lundberg

Sweden

Eve Maubec

France

David Nelson

USA

Emiko Noguchi

Japan

Rei Ogawa

Japan

Anthony Ormerod

United Kingdom

Tae Hwan Park

South Korea

Correspondence: guangdetu@biomedcentral.com

Room 1006-1007 Financial Plaza, No. 333 Jiujiang Road, Huangpu District,

Shanghai 200001, China

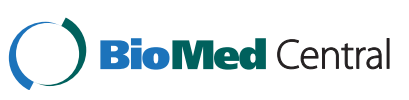

(c) 2015 Tu; licensee BioMed Central. This is an Open Access article distributed under the terms of the Creative Commons Attribution License (http://creativecommons.org/licenses/by/4.0), which permits unrestricted use, distribution, and reproduction in any medium, provided the original work is properly credited. The Creative Commons Public Domain Dedication waiver (http://creativecommons.org/publicdomain/zero/1.0/) applies to the data made available in this article, unless otherwise stated. 
Stefano Piaserico

Italy

Andor Pivarcsi

Sweden

Gaelle Quereux

France

Kristian Reich

Germany

June Robinson

USA

Laura Sawyer

United Kingdom
Fabiana Schuelter-Trevisol

Brazil

Daniel Shin

USA

Gregory Sonnenberg

USA

H. Peter Soyer

Australia

Wim Stevens

Belgium

Makoto Sugaya

Japan
Michael Sweeney

USA

Brian Tait

Australia

Andrés Tirado-Sánchez Mexico

Hywel Williams

United Kingdom

Miriam Wittmann

United Kingdom

Xianyong Yin

China 